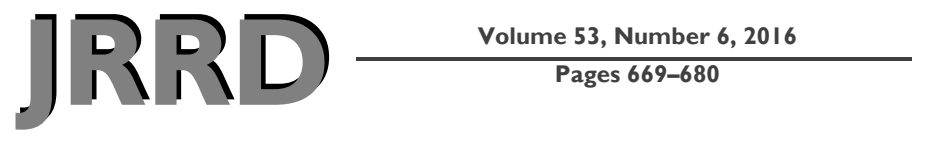

\title{
Caring for our wounded warriors: A qualitative examination of health- related quality of life in caregivers of individuals with military-related
} traumatic brain injury

\author{
Noelle E. Carlozzi, PhD; ${ }^{1 *}$ Tracey A. Brickell, DPsych; ${ }^{2-3}$ Louis M. French, PsyD; ${ }^{2-4}$ Angelle Sander, PhD; ${ }^{5}$ \\ Anna L. Kratz, PhD; ${ }^{1}$ David S. Tulsky, PhD; ${ }^{6}$ Nancy D. Chiaravalloti, PhD; ${ }^{7}$ Elizabeth A. Hahn, MA; ${ }^{8}$ Michael \\ Kallen, PhD; ${ }^{8}$ Amy M. Austin, BS; ${ }^{1}$ Jennifer A. Miner, MBA; ${ }^{1}$ Rael T. Lange, PhD $^{2,9}$ \\ ${ }^{1}$ Department of Physical Medicine and Rehabilitation, University of Michigan Medical School, Ann Arbor, MI; ${ }^{2}$ Defense \\ and Veterans Brain Injury Center and Walter Reed National Military Medical Center, Bethesda, MD; ${ }^{3}$ Uniformed Ser- \\ vices University of the Health Sciences, Bethesda, MD; ${ }^{4}$ Center for Neuroscience and Regenerative Medicine, Bethesda, \\ MD; ${ }^{5}$ Brain Injury Research Center, TIRR Memorial Hermann and Department of Physical Medicine and Rehabilitation, \\ Baylor College of Medicine, and Harris Health System, Houston, TX; ${ }^{6}$ Department of Physical Therapy, University of \\ Delaware, Newark, DE; ${ }^{7}$ Neuropsychology and Neuroscience Laboratory, Traumatic Brain Injury Laboratory, Kessler \\ Foundation Research Center, West Orange, NJ; ${ }^{8}$ Department of Medical Social Sciences, Northwestern University, Chi- \\ cago, IL; ${ }^{9}$ University of British Columbia, Vancouver, BC, Canada
}

\begin{abstract}
The purpose of this study was to develop a conceptual framework that captures aspects of health-related quality of life (HRQOL) for caregivers of individuals with military-related traumatic brain injury (TBI). We analyzed qualitative data from nine focus groups composed of caregivers of wounded warriors with a medically documented TBI. Focus group participants were recruited through hospital-based and/or community outreach efforts at the Walter Reed National Military Medical Center, the University of Michigan, and Hearts of Valor support groups (Tennessee and Washington). Participants were the caregivers $(n=45)$ of wounded warriors who had sustained a mild, moderate, severe, or penetrating TBI. Qualitative frequency analysis indicated that caregivers most frequently discussed social health (44\% of comments), followed by emotional (40\%) and physical health (12\%). Areas of discussion that were specific to this population included anger regarding barriers to health services (for caregivers and servicemembers), emotional suppression (putting on a brave face for others even when things are not going well), and hypervigilance (controlling one's behavior/environment to prevent upsetting the servicemember). Caring for wounded warriors with TBI is a complex experience that positively and negatively affects HRQOL. While some aspects of HRQOL can be evaluated with existing measures, evaluation tools for other important components do not exist. The development of military-specific measures would help facilitate better care for these individuals.
\end{abstract}

Key words: caregiver, health-related quality of life, outcome assessment (health care), quality of life, rehabilitation, servicemembers, traumatic brain injury, underserved populations, Veterans, wounded warriors.

\section{INTRODUCTION}

Caregivers of individuals with military-related traumatic brain injury (TBI) are an underserved population [1]. TBI is a common injury among military servicemembers,

Abbreviations: DOD = Department of Defense, HRQOL = health-related quality of life, $\mathrm{SD}=$ standard deviation, $\mathrm{TBI}=$ traumatic brain injury, VA = Department of Veterans Affairs.

*Address all correspondence to Noelle E. Carlozzi, PhD; Department of Physical Medicine and Rehabilitation, University of Michigan Medical School, North Campus Research Complex, Building 14, G216, 2800 Plymouth Rd, Ann Arbor, MI 48109-2800; 734-763-8917; fax: 734-7637186. Email: carlozzi@med.umich.edu http://dx.doi.org/10.1682/JRRD.2015.07.0136 
including those returning from combat operations (e.g., Operations Iraqi Freedom and Enduring Freedom) and those injured through training, accidents, and other means [2-3]. From 2000 through 2015, more than 325,000 servicemembers were diagnosed with TBI (1.5\% penetrating injuries, $1.0 \%$ severe, $8.5 \%$ moderate, $82.4 \%$ mild, and $6.6 \%$ unclassifiable) [4]. A better understanding is needed to determine how family members' health-related quality of life (HRQOL) is affected by having to provide care for a servicemember who sustains a TBI. HRQOL represents a multidimensional construct reflecting the effect of a disease, disability, or its treatment on mental, physical, and social well-being [5]. This can be contrasted with the more general term of "quality of life," which also encompasses general well-being [6-7] but lacks a consensus definition.

The limited research examining caregivers of servicemembers with TBI suggests that these caregivers experience less family cohesion and nurturance, greater emotional distress and mental health problems, and greater financial strain than their civilian counterparts [813]. These difficulties are in addition to the burdens related to deployment and reintegration (e.g., high divorce rates and family disruption) [14-16]. Servicemembers with TBI are also more likely than civilians with TBI to experience repeat injuries as well as comorbid physical and psychological ailments simultaneous with the TBI (i.e., polytrauma) [14-15]. Polytrauma occurs when an individual experiences concurrent injuries to multiple body parts and organ systems; this can include major limb trauma (single or multiple amputations), musculoskeletal injuries, burns, spinal cord injury, auditory and visual impairment, and facial disfigurement. These injuries can result in chronic pain, sexual dysfunction, substance misuse, or other problems in addition to combat-related mental health problems (e.g., posttraumatic stress disorder), all of which may make long-term caregiving more complicated [17-27].

In order to address this underserved population, Congress mandated the development of a family caregiver curriculum-Traumatic Brain Injury: A Guide for Caregivers of Service Members and Veterans-to improve educational support and to increase attention to the respite needs of caregivers of patients with TBI; it is intended for family caregivers of servicemembers and Veterans who have sustained a moderate or severe TBI [1 (Sect 744)]. It is commonly provided to family caregivers by case managers and clinicians from the Departments of Defense (DOD) and Veterans Affairs (VA).
In addition to the caregiver curriculum, the United Health Foundation funded the 2010 report Caregivers of Veterans-Serving on the Homefront [27]. This report identified caregivers of wounded servicemembers (regardless of injury etiology) as often experiencing marital strain, decreased personal time, and feelings of isolation. Relative to their civilian caregiver counterparts, caregivers of wounded servicemembers reported remaining in the caregiver role for a longer period of time and having greater physical strain, as well as higher levels of emotional stress, inability to work, and financial burden.

Furthermore, in 2006, Congress mandated that family members of a servicemember who had incurred a TBI be one of the primary DOD research focuses for Operations Iraqi Freedom and Enduring Freedom servicemembers [1 (Sec 721)]. This mandate resulted in a 15 yr longitudinal study examining the long-term effects of TBI on both the surviving servicemember and his/her family system. Several authors of this article (Brickell, French, and Lange) are currently leading several research projects addressing this mandate.

Although many of our wounded warriors receive high-quality medical care for service-related injuries, the caregivers and families of these individuals often do not receive the same level of attention. Therefore, the purpose of the current study is to identify and characterize salient domains of HRQOL that are important to caregivers of servicemembers with military-related TBI. This work should help identify the most appropriate areas for clinical interventions targeted at improving the lives of both the caregiver and the servicemember/Veteran with TBI. Furthermore, this analysis will serve as the foundation for the development of a new measurement system designed to capture the most salient aspects of HRQOL for these caregivers. This measurement system can be used to sensitively examine the interrelationships among caregiver and care-recipient HRQOL and serve as an end point for clinical trials targeted at improving the lives of these servicemembers and their families.

\section{METHODS}

\section{Participants}

Focus groups were conducted with caregivers of individuals with military-related TBI (mild, moderate, severe, and penetrating) in order to identify the most important and relevant HRQOL domains for TBI caregivers. Nine focus groups were conducted that included a total of 45 
unique caregivers of servicemembers and Veterans recruited through hospital-based and nationwide community outreach procedures at Walter Reed National Military Medical Center (Bethesda, Maryland), the University of Michigan (Ann Arbor, Michigan), and Hearts of Valor-a community organization designed to support caregivers of wounded warriors (medical documentation is required to join this organization; Tennessee and Washington). Inclusion criteria were that caregivers be at least $18 \mathrm{yr}$ old; be able to read and understand English; and be caring for an individual with a medically documented mild, moderate, or severe and/or penetrating TBI (the individual with the TBI was required to be $\geq 1$ yr postinjury and meet DOD criteria for a mild, moderate, severe, or penetrating TBI; Table 1) [28]. Medical record documentation was required to support the TBI diagnosis. Caregivers could be providing care for someone with or without comorbid bodily injuries. The caregiver role was confirmed using the following question: "On a scale of $0-10$, where 0 is 'no assistance' and 10 is 'assistance with all activities,' how much assistance does the person you care for require from you to complete activities of daily living due to problems resulting from his/her TBI? Activities could consist of personal hygiene, dressing and undressing, housework, taking medications, managing money, running errands, shopping for groceries or clothing, transportation, meal preparation and cleanup, remembering things, etc." Caregivers indicating responses of $\geq 1$ were eligible for participation in this study. Data were collected in accordance with local institutional review boards, and informed consent was provided by participants prior to participation.

Table 2 provides demographic data for study participants. Caregivers were typically in their thirties (average age $=37.3 \mathrm{yr}$; standard deviation $[\mathrm{SD}]=9.6$ ) and primarily female (93\%) and Caucasian (84\%); 9 percent were Hispanic/Latino. On average, caregivers reported serving in their caregiver role for $4.4 \mathrm{yr}(\mathrm{SD}=2.5$, range $0.6-10$ ). With regard to relationship to the servicemember, the majority of caregivers were spouses (73\%), followed by children (16\%), other relationships (male partner, fiancé, brother: $7 \%$ ), and parents (4\%). Servicemembers were $34.6 \mathrm{yr}$ of age on average (SD =6.4); 67 percent served in the Army, 11 percent Marine Corps, 7 percent Army National Guard, 4 percent Air Force, 4 percent Army Reserves, 4 percent Navy, and 2 percent Navy Reserves. In addition, 9 percent of the servicemembers were Active Duty (medical board pending; missing for 5 people). With regard to TBI severity, 49 percent were mild injuries, 13 percent moderate, 7 percent severe, and 11 percent were penetrating injuries (these data are unavailable for $20 \%$ of our participants). Sixty-seven percent of the TBIs were blast-related injuries (i.e., primary, secondary, and tertiary blast), and 33 percent were not blast-related. Forty-two percent of the injuries occurred as a part of a motor vehicle accident, 16 percent were from gunshot wounds, 13 percent were a result of the servicemember being thrown against an object, 7 percent were from falls, 7 percent were a result of the servicemember being struck by an object, 9 percent indicated more than one injury, 2 percent reported other sources of injury (e.g., improvised explosive device, rocket fire), and 4 percent were unknown. The majority of servicemember TBIs were sustained during combat deployment (69\% were combatrelated, and $13 \%$ were non-combat related). Of the remaining, 13 percent were not deployed at the time of injury, and 4 percent selected more than one option (indicative of multiple TBIs).

\section{Data Collection and Analysis}

Focus groups were about 90 min in length and led by one to two female moderators with extensive experience conducting focus groups (at least one comoderator was

Table 1.

Departments of Defense and Veterans Affairs classification for traumatic brain injury documentation.

\begin{tabular}{lccc}
\hline \multicolumn{1}{c}{ Criteria } & Mild $^{*}$ & Moderate & Severe \\
\hline Structural Imaging & Normal & Normal or abnormal & Normal or abnormal \\
Loss of Consciousness & $0-30 \mathrm{~min}$ & $>30 \mathrm{~min}$ and $<24 \mathrm{~h}$ & $>24 \mathrm{~h}$ \\
Alteration of Consciousness/Mental State & $>24 \mathrm{~h}$ \\
Posttraumatic Amnesia & $0-24 \mathrm{~h}$ & $>24 \mathrm{~h}$ & $>7 \mathrm{~d}$ \\
Glasgow Coma Scale (best available in first 24 h) & $0-1 \mathrm{~d}$ & $>1$ and $<7 \mathrm{~d}$ & $<9$ \\
\hline
\end{tabular}

*Abnormal structural imaging (e.g., magnetic resonance imaging or computed tomography scanning) attributed to the injury will result in the individual being considered clinically to have greater than mild injury.

${ }^{\dagger}$ Alteration of mental status must be immediately related to the head trauma. Typical symptoms include looking/feeling dazed, confusion, difficulty thinking clearly or responding appropriately to mental status questions, and being unable to describe events immediately before or after the trauma event. 
Table 2.

Demographic data for traumatic brain injury (TBI) caregiver participants $(N=45)$.

\begin{tabular}{|c|c|}
\hline Variable & $\begin{array}{c}\% \text { or Mean } \pm \\
\text { Standard Deviation }\end{array}$ \\
\hline Age, yr & $37.3 \pm 9.6$ \\
\hline Sex: Female & 93 \\
\hline Ethnicity: Hispanic or Latino & 9 \\
\hline Race: Caucasian & 84 \\
\hline \multicolumn{2}{|l|}{ Relationship to Servicemember } \\
\hline Spouse & 73 \\
\hline Child & 16 \\
\hline Parent & 4 \\
\hline Other & 7 \\
\hline Time in Caregiver Role, yr & $4.4 \pm 2.5$ \\
\hline Servicemember Age, yr & $34.6 \pm 6.4$ \\
\hline \multicolumn{2}{|l|}{ TBI Severity } \\
\hline Mild & 49 \\
\hline Moderate & 13 \\
\hline Severe & 7 \\
\hline Penetrating & 11 \\
\hline Unknown & 20 \\
\hline \multicolumn{2}{|l|}{ Service Branch ${ }^{*}$} \\
\hline Army & 67 \\
\hline Marine Corps & 11 \\
\hline Army National Guard & 7 \\
\hline Air Force & 4 \\
\hline Army Reserves & 4 \\
\hline Navy & 4 \\
\hline Navy Reserves & 2 \\
\hline Active Duty: Yes & 9 \\
\hline Blast-Related Injuries: Yes & 67 \\
\hline \multicolumn{2}{|l|}{ Mechanism of Injury } \\
\hline Motor Vehicle Accident & 42 \\
\hline Gunshot Wound & 16 \\
\hline Thrown Against Object & 13 \\
\hline Fall & 7 \\
\hline Struck By Object & 7 \\
\hline Multiple Mechanisms & 9 \\
\hline Other & 2 \\
\hline Unknown & 4 \\
\hline \multicolumn{2}{|l|}{ Deployment-Related Injury ${ }^{*}$} \\
\hline Yes: Combat-Related & 69 \\
\hline Yes: Non-Combat-Related & 13 \\
\hline No: Not Deployment-Related & 13 \\
\hline >1 Option (multiple injuries) & 4 \\
\hline
\end{tabular}

always a PhD-level clinical psychologist; Carlozzi or Kratz). Moderators utilized broad, open-ended questions to allow participants to articulate how being a caregiver has affected their overall HRQOL (see Appendix for the Focus Group Interview Guide, available online only). Follow-up prompts included open-ended questions designed to solicit what aspects of life had been most affected and prompts to discuss social, emotional, physical, and cognitive health. Focus groups were audiorecorded, transcribed verbatim, and de-identified. Frequency analysis [29-31] was employed according to established methodology using NVIVO 10 software (AQSR; Burlington, Massachusetts) [32-35]. This methodology utilizes the development of a domain framework (conceptual model), open and axial coding, selective coding, and descriptive analysis [34]. Three project team members (Carlozzi, Kratz, or Brickell) collaboratively identified major content areas and a list of subdomains through transcript review (open coding) and reconciled these content domains with the codebooks that were used to analyze the civilian-caregiver data [36]. This information was then used to develop a hierarchical taxonomy for each major content area. The 12 members of the investigator team ( 9 with $\mathrm{PhDs}, 2$ with master's degrees, and 1 with a bachelor's degree) with expertise in caregivers of civilians with TBI, caregivers of individuals with military TBI, patient-reported outcome measurement development, civilian TBI, and military TBI, reviewed and modified the codebooks using an iterative process. This resulted in separate codebooks for four major content areas: mental health, physical health, social health, and military-related services. Each transcript was analyzed separately according to each codebook. Thus, the same text could be coded multiple times according to the different codebooks. Prior to coding, raters (bachelor's or master's level assistants who were supervised by a PhDlevel psychologist) were required to establish interrater reliability (i.e., $\geq 80 \%$ agreement). Once interrater reliability was established, two coders coded each transcript independently; the two raters were required to establish consensus on any discrepancies that were identified during the coding review process.

\section{RESULTS}

Saturation (i.e., the point at which no new information was obtained) was achieved by the eighth focus 
group. In accordance with established qualitative methodology [34], an additional focus group was conducted after saturation to ensure that overall frequency counts were an accurate representation of the cohort. Frequency analysis percentages reflect the number of times a topic was coded relative to the total number of codes; thus, this percentage provides an indication of the relative importance of each of the domains. Qualitative frequency analysis indicated that caregivers spent the most time discussing concerns with social health $(44 \%$ of comments), followed closely by emotional health (40\%) then by physical health (12\%) and cognitive health (3\%). A detailed breakdown of the thematic content within each domain is summarized here and presented in Table 3.

\section{Social Health}

Caregivers most frequently discussed how being in a caregiving role has negatively affected their social health (44\% of the discussion). Common concerns included trouble with finances, having to give up a career to serve as a caregiver for their servicemember (vocation), how being a caregiver has changed their social roles with both the person with the TBI and others (caregiver social role change), and how this change has also affected other social systems (including other family and friend relationships). Specifically, within the military system, many caregivers receive (or were applying for) caregiver stipends that allow them to be paid for providing care for the servicemember. This type of benefit generally precludes them from having another vocation and often results in financial strain because the caregiver stipend is almost always less money than the wages an individual would be able to garner if competitively employed. In addition, much of the discussion revolved around a change in social roles or social systems. Common themes among changes in social roles included having difficulty switching back and forth from the role of a spouse to that of a caregiver ("I didn't anticipate being his parent and the next day-hour, I'm his spouse”), being uncertain of how hard to push the person with the injury ("It's a struggle between how much you do and how much you don't do"), having to manage another person who was previously independent ("I think it's hard to be like a brain for someone else"), and having to reprioritize and reevaluate what things were important ("I think that we've lowered our standards so low for a quality of life"). With regard to changing systems, common themes included family disruption ("has affected our family in ways that I had no idea it could"), being unable to do the things that were originally planned ("I feel this is not the place that I should be in at this time in my life"), and loss of friends ("I lost a whole circle of friends").

\section{Emotional Health}

Caregivers also discussed how the caregiving role has affected their emotional health (40\% of the discussion). The most commonly discussed concerns included caregiver strain/burden, feelings of loss (grief over things that have changed for the servicemember because of the injury, as well as grief related to self-sacrifice and having to give up things to care for the servicemember), feelings of anger (much of which was anger/frustration with the military and VA healthcare systems), depression, and anxiety. These caregivers also frequently discussed experiencing anxiety that was focused on having to be hypervigilant. For example, caregivers expressed having to constantly monitor their own behavior, the behavior of other individuals, and the behavior of the servicemember in order to minimize upsetting their servicemember. Failure to do so might result in emotionally upsetting their servicemember or even physical violence (directed at the caregiver or at other individuals). One caregiver commented, "We're constantly on guard of anywhere you go to eat or anywhere you go out . . . that it's like okay, is this place going to be good for him?”

Furthermore, caregivers frequently discussed living within the military culture and how there were both internal and external pressures to maintain the appearance that things were okay even if they are not ("We're taught . . . you don't break down. So a lot of our emotions are hidden”). Positive aspects of being a caregiver were also discussed. There was a general feeling of pride and respect for the sacrifices that their loved one had made for their country; being able to care for this individual fostered individual pride ("We're proud to be able to serve this way ... just because he's not serving in the same way ... doesn't mean that we're not still serving our country”).

\section{Physical/Medical Health}

Issues and concerns about changes in physical health were less common (12\% of the discussion). Nonetheless, discussion of health behaviors (including lifestyle choices such as diet and exercise), as well as difficulties keeping up with their own medical care, was a predominant focus within this domain. For some, the injury also negatively affected their intimacy/sexual life with their partners 
JRRD, Volume 53, Number 6, 2016

Table 3.

Health-related quality of life (HRQOL) themes generated among caregivers of individuals with a military-related traumatic brain injury (TBI).

HRQOL Domain and Subdomains
Example Quotation
Results from

Overall Thematic Breakdown (\%)
Civilian TBI Caregivers (\%) [36]

\begin{tabular}{|c|c|}
\hline 44 & 42 \\
\hline 17 & 9 \\
\hline 15 & $31^{*}$ \\
\hline 13 & $31^{\dagger}$ \\
\hline 11 & $31^{*}$ \\
\hline 9 & 7 \\
\hline 8 & $31^{\dagger}$ \\
\hline 6 & $31^{\dagger}$ \\
\hline 5 & 7 \\
\hline 5 & 5 \\
\hline 3 & $31^{\dagger}$ \\
\hline 3 & 6 \\
\hline 40 & 34 \\
\hline 20 & 22 \\
\hline 13 & $9^{\ddagger}$ \\
\hline 12 & 10 \\
\hline 10 & 6 \\
\hline 9 & 7 \\
\hline 7 & 5 \\
\hline 7 & - \\
\hline 5 & - \\
\hline 5 & 6 \\
\hline 12 & 11 \\
\hline 20 & 27 \\
\hline 15 & 20 \\
\hline 14 & 6 \\
\hline 12 & 8 \\
\hline 7 & 10 \\
\hline 4 & 5 \\
\hline 2 & 3 \\
\hline 4 & 3 \\
\hline
\end{tabular}

\author{
Emotional Support \\ Community Life/Recreation \\ Stigma
}

Companionship

\section{Isolation}

Emotional Health

Caregiver Strain

Feelings of Loss

Anger

Depression/Self-Esteem

Positive Emotions

General Anxiety

Hypervigilance

Suppression

Emotional Roadblocks

Physical Health

Health Behaviors

Medical Care/Medication

Sexual Functioning

Fatigue

Sleep

Pain

Gastrointestinal Complaints

Cognitive Health
“And I mean, even at 100\% rating, and getting your caregiver stipend, if you own a home and you have a car payment and you have bills, you're not making ends meet."

"And most of the time I don't see him as my husband. I see him as a child. As one of the kids."

"There's just not a lot of-I mean the resources are out there, but they're not very helpful when you call them."

"We've probably all lost a lot of close connections with family."

"So I had to leave my job and become his full-time caregiver."

"But most people don't realize that, okay, yes, helping once a week, that's awesome, but sometimes there's people that need help like every single day.”

"But there's nobody for me as a caregiver to turn to."

"I'm so proud to be part of the wounded warrior community."

"Or we can go somewhere without my husband. But they don't allow their children around my husband, even though they've never had any kind of experience or you know."

"Yeah. And it's always, you know if I want to cuddle on the couch, he wants nothing to do with me."

"I feel isolated. I feel isolated a lot."

"It's just you kind of almost feel like you're drowning trying to help them."

"I think for my son, I just-it breaks my heart that at age 20 he had this horrible injury and his life will never be the same."

"I feel a lot of anger and resentment."

"I mean it's hard, I think, not to be depressed when you're dealing with some of these things."

"I’m very grateful every day I have my husband."

"I have some anxiety issues and it all relates around his behavior."

"We're very guarded—on guard a lot just to make sure that they're okay."

"How do you feel? That's a question I get a lot. How do you feel? I try not to."

"But I feel like I can’t be honest about what my needs are.”

“And it really wasn't even up [until] like 2 years ago—we're 8 years into this- that I started to do more work on myself, for my health."

"I was just put on depression meds."

"Like you know, I don't have those sexual thoughts in my head anymore."

"But then we're also so exhausted at the end of each day."

"I don’t sleep as well as I used to."

"I wake up in the morning with my jaw hurting because I’m just so tense."

"Because I have a stomach condition."

"My memory issues are very, very problematic."
Note: Percentages for domains (boldface) reflect the total percentage of comments related to this specific domain (domain percentages should sum to 100 with rounding); percentages within each subdomain reflect the percentage of comments within each domain (should sum to $<100$ since content that did not fit well under a specific subdomain category would have only been counted in the domain summary). Results from civilian caregiver groups are published elsewhere [36] and are shown here for comparison purposes.

${ }^{*}$ Civilian analyses for role changes combine military concepts of caregiver social role change and social system change (for comparison, overall percentages for these subdomains are repeated in this table).

${ }^{\dagger}$ Civilian analyses for companionship included the concepts of companionship, informational support, emotional support, and instrumental support from the military analysis (for comparison, overall percentages for this subdomain are repeated in this table).

${ }^{\ddagger}$ Civilian analyses for feelings of loss were coded differently than the military analysis—-for the civilian analysis, this construct was coded as a separate, stand-alone content area; for the military analysis this construct was coded as a subcomponent of emotional health. 
("There's no . . . intimacy left because he's not able to even really communicate with me"). Fatigue and sleep difficulties were also common complaints.

\section{Cognitive Health}

Cognitive concerns were only occasionally discussed and most likely were raised in response to direct inquiries from the moderators ( $4 \%$ of the discussion). Cognitive concerns were most frequently articulated as not feeling as "sharp" as he or she used to be (because of being overwhelmed by the caregiver role) and feeling like he or she had TBI via proxy ("It's a joke in the caregiver community that TBI is contagious").

\section{DISCUSSION}

This study highlights the different aspects of HRQOL that are affected by caring for a servicemember or Veteran with TBI. With regard to social functioning, caregivers expressed concerns about finances (the financial impact of having to give up a vocation to become a paid caregiver for their loved one) and how TBI has resulted in changes in their social system (affecting the relationships they have with their servicemember), as well as the changes this had on the larger social network (including other family and friend relationships). All of these social concerns generally overlap and are consistent with those that were raised in similar focus groups in a civilian cohort [36]. With regard to finances and financial strain, the military caregivers frequently discussed military-provided caregiver compensation; these caregivers lived in constant fear of losing these benefits. On the other hand, civilian caregivers do not have comparable caregiver stipend benefits (although caregivers may receive monetary compensation through Medicaid; this was not raised as part of the civilian caregiver discussion). Discussion for civilian caregivers (with regard to finances and financial strain) focused more on loss of work (for both the caregiver and individuals with the TBI), as well as on how the medical expenses related to TBI treatment and rehabilitation affected them.

In addition to the social concerns that were raised, emotional concerns included caregiver strain/burden, feelings of loss, depression, and general anxiety, all of which were consistent with concerns raised by caregivers of civilians with TBI [36]. However, in contrast to civilian caregivers, caregivers of military TBI also frequently discussed concerns of hypervigilance or having to con- stantly monitor and control the environment so as to avoid people and situations that might upset their servicemembers. This concern was unique to military caregivers relative to civilian caregivers, who minimally discussed this topic [36]. We postulate that this difference (in military vs civilian caregivers) is likely due to the high rates of comorbid posttraumatic stress disorder in this population [37]. Mild/prodromal posttraumatic stress symptomatology may be more pronounced in individuals with comorbid TBI. Specifically, individuals with comorbid TBI and posttraumatic stress may have a harder time coping with and managing the stressors associated with combat-related deployment(s). These individuals may also have a harder time readjusting to civilian life or may have cognitive limitations that limit full participation in some psychotherapeutic modalities. Caregivers of servicemembers with TBI also placed greater emphasis on emotional suppression (i.e., having to hide what they are really feeling) relative to their civilian counterparts [36]. This is consistent with work highlighting the unique aspects of military culture [38-39] and how any admitted weakness may result in a significant reduction in duties and responsibilities [39].

Caregivers of servicemembers with TBI also expressed a lot of anger. Although civilian caregivers also express anger [36], the qualitative nature of this anger was different. Specifically, caregivers of military TBI articulated anger related to perceived lack of availability, accessibility, or ease of navigating the military or VA healthcare system. Difficulty qualifying for appropriate benefits, getting timely appointments, and disruption of financial benefits were common complaints and significant sources of anger for many caregivers. A lack of coordinated care for the caregiver and/or other family members was also a frequent source of caregiver anger. These complaints highlight areas where future improvements in accessibility or care navigation may be beneficial.

Finally, caregivers of servicemembers with TBI also talked about the effects that caregiving has on both physical and cognitive health. Similar to caregivers of civilians with TBI [36], these military caregivers discussed health behaviors, difficulty keeping up with their own medical care, changes in their sex lives, and problems with sleep and fatigue, as well as some limited discussion of how the stress of caring for someone negatively affects cognition.

While this work highlights the many effects that caring for someone with a military-related TBI can have on 
HRQOL, there are some study limitations. First, the majority of caregivers were spouses. We have previously discovered differences between parent and spouse caregivers of civilians with TBI [40], and differences by relationship type should be explored in caregivers of military TBI. We also did not examine the impact that length of time providing care had on the caregiver role. We also did not collect data on where the caregiver/servicemember was receiving care (i.e., DOD or VA healthcare system); therefore, we are unable to draw specific conclusions about the origins of specific healthcare complaints. In addition, although we required medical documentation of TBI for the injured servicemember, we did not consistently have enough medical record documentation to confirm TBI severity (this was missing for $20 \%$ of our sample). Thus, future work is needed to examine the relationship between TBI severity and caregiver HRQOL. In addition, we did not consistently collect information about comorbidities. For example, although we assume that the comorbid rates of TBI and posttraumatic stress disorder are comparable to general rates within these returning servicemembers, we do not have enough information to characterize this in our sample. Future work should examine how these common comorbidities and/or polytrauma affect HRQOL for these caregivers. Furthermore, although comparisons were made between civilian and military caregivers, inclusion criteria for these study samples were not identical. In particular, civilian caregivers had to be providing care for an individual with a moderate or severe TBI [41], whereas military caregivers had to be providing care for an individual with a DOD-defined TBI (which includes mild TBI) [28]. This deliberate departure for our military caregivers reflects the fact that the majority of military-related TBIs are of mild severity (i.e., 82.4\%) [4]; to exclude mild TBI from a military sample would minimize the generalizability of findings for this population. Thus, differences among civilian and military caregivers may be due in part to the systematic differences between the TBI groups for whom these individuals are providing care. We also did not control for interviewer bias in our study. Finally, we employed a qualitative approach that was designed specifically for the development of new patient-reported outcomes measures and relies more heavily on the relative importance of specific topic areas, rather than more traditional qualitative analysis approaches. Future analysis using these more traditional qualitative analysis approaches are underway and may yield a different pattern of findings; these more traditional approaches might highlight important differences for a number of the different demographic variables that were evaluated for our sample (e.g., respondent type, length of time in a caregiver role, how personal values interface with HRQOL).

\section{CONCLUSIONS}

To our knowledge, this is the first study to employ a qualitative focus group methodology to examine HRQOL for caregivers of individuals with military TBI. While this work highlights a number of HRQOL issues that are common to both caregivers of servicemember TBI and caregivers of civilian TBI, it also highlights a number of factors that are unique to the military caregiver experience. In particular, caregiver hypervigilance, emotional suppression, and anger with regard to ease of access of military and/or VA healthcare or financial services are areas that warrant future investigation and intervention. Future work is needed to address the complicated issues that face these caregivers and the servicemembers for whom they provide care. To this end, we are in the process of developing a new set of measures that captures these aspects of HRQOL for these caregivers. Ultimately, such work should target improving HRQOL for these servicemembers and their families.

\section{ACKNOWLEDGMENTS}

\section{Author Contributions:}

Study principal investigator; oversight for qualitative analysis; initial draft of Introduction, Methods, Results, and Discussion; and incorporation of revisions: N. E. Carlozzi.

Study co-investigator: A. Sander, A. L. Kratz, D. S. Tulsky, N. D. Chiaravalloti, E. A. Hahn, M. Kallen.

Military study co-investigator: T. A. Brickell, L. M. French, R. T. Lange. Data collection site: N. E. Carlozzi, T. A. Brickell, L. M. French, R. T. Lange.

Codebook development: N. E. Carlozzi, A. L. Kratz.

Codebook refinement: T. A. Brickell, L. M. French, A. Sander,

D. S. Tulsky, N. D. Chiaravalloti, E. A. Hahn, M. Kallen, J. A. Miner, R. T. Lange.

Coding reconciliation: A. L. Kratz.

Primary coder, responsible for qualitative analysis, assistance with Methods and Results, and study research assistant: A. M. Austin. Draft of study protocol for Methods and study project manager: J. A. Miner.

Focus group moderator: N. E. Carlozzi, A. L. Kratz.

Developed methodology for qualitative analysis: D. S. Tulsky. Assistance with writing the Introduction and Discussion: T. A. Brickell, R. T. Lange. 
Review and feedback on manuscript drafts: T. A. Brickell, L. M. French, A. Sander, A. L. Kratz, D. S. Tulsky, N. D. Chiaravalloti, E. A. Hahn, M. Kallen, A. M. Austin, J. A. Miner, R. T. Lange.

Financial Disclosures: The authors have declared that no competing interests exist.

Funding/Support: This material was based on work supported by the National Institutes of Health, National Institute of Nursing Research (grant R01-NR013658). This material is also based upon work supported in part by the Defense and Veterans Brain Injury Center, U.S. Army Medical Research and Materiel Command. Dr. Kratz's contribution to this manuscript was supported, in part, by the National Institutes of Health, National Institute of Arthritis and Musculoskeletal and Skin Diseases (grant K01AR064275).

Institutional Review: Data were collected in accordance with local institutional review boards; informed consent was provided by participants prior to participation.

Participant Follow-Up: The authors do not plan to inform participants of the publication of this study. However, participants receive a newsletter of study updates that will encourage them to check our Web site for updated publications.

Disclaimer: The views, opinions, and/or findings contained in this article are those of the authors and should not be construed as an official DOD position, policy, or decision unless so designated by other official documentation.

\section{REFERENCES}

1. John Warner National Defense Authorization Act for Fiscal Year 2007, Pub. L. 109-364, 120 Stat. 2083 (Oct. 17, 2006).

2. Fischer H. A guide to U.S. military casualty statistics: Operation Freedom's Sentinel, Operation Inherent Resolve, Operation New Dawn, Operation Iraqi Freedom, and Operation Enduring Freedom. Washington (DC): Congressional Research Service (US); 2015 Aug 7. 10 p. Report No.: RS22452.

3. Bagalman E. Traumatic brain injury among veterans. Washington (DC): Congressional Research Service (US); 2013 Jan 4. 20 p. Report No.: R40941.

4. Defense and Veterans Brain Injury Center (DVBIC). DOD worldwide numbers for TBI [Internet]. Silver Spring (MD): DVBIC; [cited 2015 Feb 17]. Available from: http://dvbic.dcoe.mil/dod-worldwide-numbers-tbi

5. Cella DF. Measuring quality of life in palliative care. Semin Oncol. 1995;22(2 Suppl 3):73-81. [PMID:7537908]

6. Campbell AJ, Converse PE, Rodgers WL. The quality of American life: Perceptions, evaluations, and satisfactions. New York (NY): Russell Sage Foundation; 1976.

7. Patrick DL, Erikson P. What constitutes quality of life? Concepts and dimensions. Clin Nutr. 1988;7(2):53-63.

8. Griffin JM, Friedemann-Sánchez G, Jensen AC, Taylor BC, Gravely A, Clothier B, Simon AB, Bangerter A, Pickett T, Thors C, Ceperich S, Poole J, van Ryn M. The invisible side of war: Families caring for US service members with traumatic brain injuries and polytrauma. J Head Trauma Rehabil. 2012;27(1):3-13. [PMID:21873883]

http://dx.doi.org/10.1097/HTR.0b013e3182274260

9. Lester P, Peterson K, Reeves J, Knauss L, Glover D, Mogil C, Duan N, Saltzman W, Pynoos R, Wilt K, Beardslee W. The long war and parental combat deployment: Effects on military children and at-home spouses. J Am Acad Child Adolesc Psychiatry. 2010;49(4):310-20. [PMID:20410724]

10. Mansfield AJ, Kaufman JS, Marshall SW, Gaynes BN, Morrissey JP, Engel CC. Deployment and the use of mental health services among U.S. Army wives. N Engl J Med. 2010;362(2):101-9. [PMID:20071699] http://dx.doi.org/10.1056/NEJMoa0900177

11. Taft CT, Schumm JA, Panuzio J, Proctor SP. An examination of family adjustment among Operation Desert Storm veterans. J Consult Clin Psychol. 2008;76(4):648-56. [PMID:18665692] http://dx.doi.org/10.1037/a0012576

12. Ruff RL, Ruff SS, Wang X-F. Improving sleep: Initial headache treatment in OIF/OEF veterans with blastinduced mild traumatic brain injury. J Rehabil Res Dev. 2009;46(9):1071-84. [PMID:20437313] http://dx.doi.org/10.1682/JRRD.2009.05.0062

13. Phelan SM, Griffin JM, Hellerstedt WL, Sayer NA, Jensen AC, Burgess DJ, van Ryn M. Perceived stigma, strain, and mental health among caregivers of veterans with traumatic brain injury. Disabil Health J. 2011;4(3):177-84.

[PMID:21723524]

http://dx.doi.org/10.1016/j.dhjo.2011.03.003

14. Dausch BM, Saliman S. Use of family focused therapy in rehabilitation for veterans with traumatic brain injury. Rehabil Psychol. 2009;54(3):279-87. [PMID:19702426] http://dx.doi.org/10.1037/a0016809

15. McFarlane AC. Military deployment: The impact on children and family adjustment and the need for care. Curr Opin Psychiatry. 2009;22(4):369-73. [PMID:19424067] http://dx.doi.org/10.1097/YCO.0b013e32832c9064

16. Riviere LA, Merrill JC, Thomas JL, Wilk JE, Bliese PD. 2003-2009 marital functioning trends among U.S. enlisted soldiers following combat deployments. Mil Med. 2012; 177(10):1169-77. [PMID:23113443] http://dx.doi.org/10.7205/MILMED-D-12-00164

17. Hoge CW, McGurk D, Thomas JL, Cox AL, Engel CC, Castro CA. Mild traumatic brain injury in U.S. Soldiers returning from Iraq. N Engl J Med. 2008;358(5):453-63. [PMID:18234750] http://dx.doi.org/10.1056/NEJMoa072972

18. Howe LL. Giving context to post-deployment postconcussive-like symptoms: Blast-related potential mild traumatic brain injury and comorbidities. Clin Neuropsychol. 2009; 23(8):1315-37. [PMID:19882474] http://dx.doi.org/10.1080/13854040903266928 
19. Iverson G, Zasler N, Lange R. Post-concussive disorder. In: Zasler N, editor. Brain injury medicine New York (NY): Demos Medical Publishing; 2007. p. 373-405.

20. Lapierre CB, Schwegler AF, Labauve BJ. Posttraumatic stress and depression symptoms in soldiers returning from combat operations in Iraq and Afghanistan. J Trauma Stress. 2007;20(6):933-43. [PMID:18157882] http://dx.doi.org/10.1002/jts.20278

21. Pietrzak RH, Johnson DC, Goldstein MB, Malley JC, Southwick SM. Posttraumatic stress disorder mediates the relationship between mild traumatic brain injury and health and psychosocial functioning in veterans of Operations Enduring Freedom and Iraqi Freedom. J Nerv Ment Dis. 2009;197(10):748-53. [PMID:19829203] http://dx.doi.org/10.1097/NMD.0b013e3181b97a75

22. Schneiderman AI, Braver ER, Kang HK. Understanding sequelae of injury mechanisms and mild traumatic brain injury incurred during the conflicts in Iraq and Afghanistan: Persistent postconcussive symptoms and posttraumatic stress disorder. Am J Epidemiol. 2008;167(12): 1446-52. [PMID:18424429] http://dx.doi.org/10.1093/aje/kwn068

23. Stein MB, McAllister TW. Exploring the convergence of posttraumatic stress disorder and mild traumatic brain injury. Am J Psychiatry. 2009;166(7):768-76. [PMID:19448186] http://dx.doi.org/10.1176/appi.ajp.2009.08101604

24. Wilkinson CW, Pagulayan KF, Petrie EC, Mayer CL, Colasurdo EA, Shofer JB, Hart KL, Hoff D, Tarabochia MA, Peskind ER. High prevalence of chronic pituitary and target-organ hormone abnormalities after blast-related mild traumatic brain injury. Front Neurol. 2012;3:11.

[PMID:22347210] http://dx.doi.org/10.3389/fneur.2012.00011

25. Frappell-Cooke W, Gulina M, Green K, Hacker Hughes J, Greenberg N. Does trauma risk management reduce psychological distress in deployed troops? Occup Med (Lond). 2010;60(8):645-50. [PMID:20889815] http://dx.doi.org/10.1093/occmed/kqq149

26. Melcer T, Sechriest VF, Walker J, Galarneau M. A comparison of health outcomes for combat amputee and limb salvage patients injured in Iraq and Afghanistan wars. J Trauma Acute Care Surg. 2013;75(2 Suppl 2):S247-54. [PMID:23883916] http://dx.doi.org/10.1097/TA.0b013e318299d95e

27. National Alliance for Caregiving/United Health Foundation. Caregivers of veterans-Serving on the homefront [Internet]. Bethesda (MD): National Alliance for Caregiving/United Health Foundation; 2010 Nov [cited 2015 Feb]. Available from:

http://www.caregiving.org/data/

2010_Caregivers_of_Veterans_FULLREPORT_WEB_FI NAL.pdf
28. Management of Concussion/mTBI Working Group. VA/ DoD Clinical practice guideline for management of concussion/mild traumatic brain injury. J Rehabil Res Dev. 2009; 46(6):CP1-68. [PMID:20108447]

29. Basch CE. Focus group interview: An underutilized research technique for improving theory and practice in health education. Health Educ Q. 1987;14(4):411-48. [PMID:3319971] http://dx.doi.org/10.1177/109019818701400404

30. Basch CE, DeCicco IM, Malfetti JL. A focus group study on decision processes of young drivers: Reasons that may support a decision to drink and drive. Health Educ Q. 1989; 16(3):389-96. [PMID:2793494] http://dx.doi.org/10.1177/109019818901600307

31. Zemke R, Kramlinger T. Figuring things out. Reading (MA): Addison-Wesley; 1985.

32. Carlozzi NE, Fyffe D, Morin KG, Byrne R, Tulsky DS, Victorson D, Lai JS, Wecht JM. Impact of blood pressure dysregulation on health-related quality of life in persons with spinal cord injury: Development of a conceptual model. Arch Phys Med Rehabil. 2013;94(9):1721-30. [PMID:23499779]

http://dx.doi.org/10.1016/j.apmr.2013.02.024

33. Carlozzi NE, Tulsky DS, Kisala PA. Traumatic brain injury patient-reported outcome measure: Identification of healthrelated quality-of-life issues relevant to individuals with traumatic brain injury. Arch Phys Med Rehabil. 2011; 92(10 Suppl):S52-60. [PMID:21958923] http://dx.doi.org/10.1016/j.apmr.2010.12.046

34. Kisala PA, Tulsky DS. Opportunities for CAT applications in medical rehabilitation: Development of targeted item banks. J Appl Meas. 2010;11(3):315-30. [PMID:20847478]

35. Carlozzi NE, Tulsky DS. Identification of health-related quality of life (HRQOL) issues relevant to individuals with Huntington disease. J Health Psychol. 2013;18(2):212-25. [PMID:22427174] http://dx.doi.org/10.1177/1359105312438109

36. Carlozzi NE, Kratz AL, Sander AM, Chiaravalloti ND, Brickell TA, Lange RT, Hahn EA, Austin A, Miner JA, Tulsky DS. Health-related quality of life in caregivers of individuals with traumatic brain injury: Development of a conceptual model. Arch Phys Med Rehabil. 2015;96(1): 105-13. [PMID:25239281] http://dx.doi.org/10.1016/j.apmr.2014.08.021

37. Coll JE, Weiss EL, Yarvis JS. No one leaves unchanged: Insights for civilian mental health care professionals into the military experience and culture. Soc Work Health Care. 2011;50(7):487-500. [PMID:21846250] http://dx.doi.org/10.1080/00981389.2010.528727

38. Hall LK. The importance of understanding military culture. Soc Work Health Care. 2011;50(1):4-18. 
[PMID:21240768]

http://dx.doi.org/10.1080/00981389.2010.513914

39. Meyer EG. The importance of understanding military culture. Acad Psychiatry. 2015;39(4):416-18. [PMID:25690349] http://dx.doi.org/10.1007/s40596-015-0285-1

40. Kratz AL, Sander AM, Brickell TA, Lange RT, Carlozzi NE. Traumatic brain injury caregivers: A qualitative analysis of spouse and parent perspectives on quality of life. Neuropsychol Rehabil. 2015;1-22. [PMID:26052805] http://dx.doi.org/10.1080/09602011.2015.1051056

41. Traumatic Brain Injury Model Systems National Data Center. Traumatic Brain Injury Model Systems [Internet]. Englewood (CO): TBI Model Systems National Data and Statistical Center; 2006. Available from: https://www.tbindsc.org/StaticFiles/Documents/ 2016\%20TBIMS\%20Slide\%20Presentation.pdf
Submitted for publication July 17, 2015. Accepted in revised form November 10, 2015.

This article and any supplementary material should be cited as follows:

Carlozzi NE, Brickell TA, French LM, Sander A, Kratz AL, Tulsky DS, Chiaravalloti ND, Hahn EA, Kallen M, Austin AM, Miner JA, Lange RT. Caring for our wounded warriors: A qualitative examination of health-related quality of life in caregivers of individuals with militaryrelated traumatic brain injury. J Rehabil Res Dev. 2016; 53(6):669-80.

http://dx.doi.org/10.1682/JRRD.2015.07.0136

ORCID: Noelle E. Carlozzi, PhD: 0000-0003-0439-9429

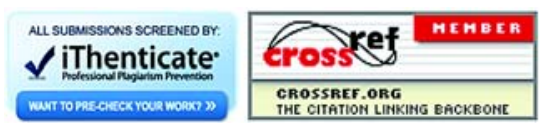


